\title{
The structure, functions and degradation of pigment-binding proteins of photosystem II
}

\author{
Robert Luciński and Grzegorz Jackowski ${ }^{\bowtie}$ \\ Department of Plant Physiology, Faculty of Biology, Adam Mickiewicz University, Poznań, Poland; \\ 凶e-mail: grzesiek@amu.edu.pl
}

Received: 11 July, 2006; revised: 25 September, 2006; accepted: 03 November, 2006 available on-line: 14 November, 2006

\begin{abstract}
Eleven proteins belonging to photosystem II (PSII) bind photosynthetic pigments in the form of thylakoid membrane-associated pigment-protein complexes. Five of them (PsbA, PsbB, PsbC, PsbD and PsbS) are assigned to PSII core complex while the remaining six (Lhcb1, Lhcb2, Lhcb3, Lhcb4, Lhcb5 and Lhcb6) constitute, along with their pigments, functional complexes situated more distantly with regard to P680 - the photochemical center of PSII. The main function of the pigment-binding proteins is to harvest solar energy and deliver it, in the form of excitation energy, ultimately to P680 although individual pigment-proteins may be engaged in other photosynthesis-related processes as well. The aim of this review is to present the current state of knowledge regarding the structure, functions and degradation of this family of proteins.
\end{abstract}

Keywords: chlorophyll-binding protein, chloroplast, core complex, light-harvesting complex, photosystem II, transmembrane $\alpha$-helices

\section{INTRODUCTION}

The vast majority of proteins of the thylakoid membrane are housed by four functional complexes embedded in these membranes: photosystem II (PSII), cytochrome $b_{6}-f$ complex, photosystem I and ATP synthase. The cooperation of the above complexes determined by the unique topology of their components as well as the lateral heterogeneity of the distribution of the complexes within the membrane facilitates the operation of photosynthetic light reactions, i.e. the interception of light energy, the migration of excitation energy toward the reaction centers of the photosystems, charge separation, electron transport, proton translocation, and photophosphorylation. An individual task of PSII is to the use the solar energy to drive the electron transport chain from water to plastoquinone, accompanied by oxygen evolution and deposition of protons in thylakoid lumen (Yamamoto, 2001).

PSII from higher plants, green algae and cyanobacteria is an enormously complicated, multisubunit particle that can be divided into two build- ing blocks believed to be organized hierarchically around a P680 molecule, i.e.

a) PSII core complex, existing as a dimer in stacked thylakoid membranes and, most probably, as a monomer in unstacked thylakoid membranes, composed of at least 25 individual proteins, two of which (PsbA and PsbD) cooperatively bind the primary chlorophyll donor P680 as well as two peripheral chlorophylls $a$ and one $\beta$-carotene along with all the cofactors mediating the electron flow $\left([\mathrm{Mn}]_{4}, \mathrm{Tyr}_{\mathrm{Z}}\right.$, pheophytin $a, \mathrm{Q}_{\mathrm{A}^{\prime}}, \mathrm{Q}_{\mathrm{B}}$ and nonheme $\mathrm{Fe}$ ) while two others (PsbB and PsbC) bind an array of chlorophyll $a$ and $\beta$-carotene molecules in the form of inner light-harvesting complexes - CP47 and CP43 (Barber et al., 1997). PbsA-F and PbsI along with their cofactors constitute a minimal subfragment of PSII core complex demonstrated to be capable of charge separation, designated PSII reaction center (Nanba \& Satoh, 1987; Ikeuchi \& Inoue, 1988).

b) six individual proteins (Lhcb1-6) binding an array of chlorophyll $a$, chlorophyll $b$, lutein, violaxanthin, zeaxanthin and antheraxanthin molecules

Abbreviations: Chl, chlorophyll; LHCII, light-harvesting complex of photosystem II; PSI, photosystem I; PSII, photosystem II. 


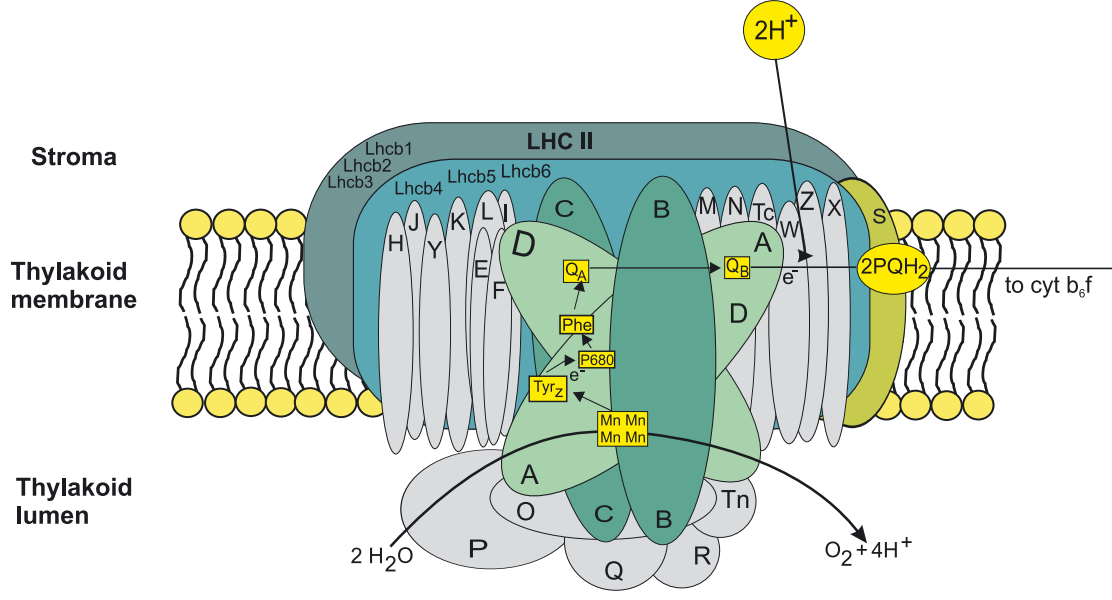

Figure 1. Schematic model of structural organization of PSII within thylakoid membrane.

Pigment-binding proteins of PSII are labelled in green. in the form of peripheral light harvesting complexes - LHCII, CP29, CP26 and CP24 (Paulsen, 1995).

Some photosynthetic pigment molecules may be bound also by the PsbS protein, classified as a PSII core complex component, although the evidence supporting this view is controversial (Funk et al., 1995; Dominici et al., 2002) (Fig. 1).

The content of this review is focused on the current understanding of the structure and functions of individual pigment-binding apoproteins of PSII (PsbA-D, PsbS, Lhcb1-6) and the supramolecular complexes formed by these proteins. A regulatory proteolysis of PSII pigment-binding proteins is also discussed.

\section{PIGMENT-BINDING PROTEINS OF PSII CORE COMPLEX}

Structure, function and degradation of pigment-binding proteins of PSII core complex

PsbA

PsbA (traditionally designated D1) is a highly conserved, pigment-binding protein of PSII reaction center (Wollman et al., 1999; Ferreira et al., 2004). The protein is encoded by a single gene localized in the chloroplast genome and synthesized in the form of a precursor containing an exceptional, C-terminal extension, which is cleaved (by CtpA protease) after insertion of pre-PsbA into the thylakoid membrane (Inagaki et al., 1996). The molecular mass of PsbA may vary depending on species but usually equals 38 kDa (Barber et al., 1997).

PsbA is an integral thylakoid membrane protein; its three-dimensional structure has been studied using electron crystallography (Rhee et al., 1998) and this study has recently been extended allowing the identification of all five transmembrane $\alpha$-helices of PsbA (designated A to E) (Hankamer et al., 2001; Ferreira et al., 2004) and two surface $\alpha$-helices
- one localized between C and D (luminal) and the other between D and E (stromal) (Michel \& Deisenhofer, 1988). The organization of the transmembrane $\alpha$-helices of PsbA is almost identical to that of the L subunit of the photosynthetic purple bacteria reaction center (Deisenhofer et al, 1985; Ferreira et al., 2004), the C-terminal domains and the loops joining the transmembrane helices are, however, more extended in PsbA than in the L subunit of purple bacteria reaction center (Ferreira et al., 2004). PsbA, together with PsbD, forms a heterodimer localized right in the middle of PSII reaction center. The heterodimer binds all the cofactors involved in the primary charge separations, water oxidation and electron flow from water to plastoquinone, namely $[\mathrm{Mn}]_{4}, \mathrm{Tyr}_{\mathrm{Z}}$, two pheophytins $a, \mathrm{Q}_{\mathrm{A}^{\prime}}$ nonheme Fe and $\mathrm{Q}_{\mathrm{B}}$ as well as six chlorophyll $a$ molecules (Ferreira et al., 2004). Four of these molecules (designated $\mathrm{Chl}_{\mathrm{D} 1}, \mathrm{Chl}_{\mathrm{D} 2}, \mathrm{P}_{\mathrm{D} 1}$ and $\mathrm{P}_{\mathrm{D} 2}$ ) together constitute what is called P680 and it is $\mathrm{P}_{\mathrm{D} 1}$ that is involved in the primary charge separation (Ferreira et al., 2004). It is bound to PsbA via His198 (Barber et al., 1997). Apart from those four chlorophylls, PsbA/PsbD heterodimer binds two peripheral chlorophyll a molecules designated $\mathrm{Chl}_{\mathrm{ZD} 1}$ and $\mathrm{Chl}_{\mathrm{ZD} 2}$, a pair stabilized additionally by PsbI and PsbX - low-molecular weight proteins of PSII core complex (Ferreira et al., 2004).

Pheophytins $a$, in turn, bind PsbA via Tyr126 and Tyr147 and $\mathrm{Q}_{\mathrm{B}}$ via interactions with His215 and Ser254 (domain B) (Ferreira et al., 2004). Potential ligands of the Mn cluster (Asp170, His190, His332, Glu333, His337, Asp342 and C-terminus of Ala344) have been identified using site-directed mutagenesis (Li \& Burnap, 2002).

The electron flow through PSII begins with the release of the electron from an excited P680 molecule when the excitation energy arrives at P680 after being transferred by peripheral and inner lightharvesting complexes as well as peripheral chlorophyll $a$ molecules $\mathrm{Chl}_{\mathrm{ZD} 1}$ and $\mathrm{Chl}_{\mathrm{ZD} 2}$ of PSII reaction center. Then the electron is transferred to $Q_{B}$ through 
pheophytin $a$ and $\mathrm{Q}_{\mathrm{A}}$ (which is bound by PsbD). After accepting two electrons and being protonated by two protons from the stroma, reduced $Q_{B}$ is released from PSII and diffuses freely as $\mathrm{PQH}_{2}$ in the lipid bilayer of thylakoid membrane toward cytochrome $b_{6}-f$ complex. The electron transfer between $Q_{A}$ and $\mathrm{Q}_{\mathrm{B}}$ is mediated by nonheme Fe which is cooperatively bound by PsbA and PsbD.

The transfer of electrons from excited P680 to pheophytin $a$ generates the cationic radical $\mathrm{P} 680^{\circ}$, which is then reduced by the redox-active tyrosine $161\left(\mathrm{Tyr}_{\mathrm{z}}\right)$ of PsbA. The resulting neutral radical $\operatorname{Tyr}_{Z}{ }^{\bullet}$ acts as an oxidant in the water oxidation process which is catalyzed by a PSII subfragment designated "oxygen evolving complex".

A very characteristic feature of PsbA is that this protein is photodamaged more rapidly under high irradiance conditions than any other protein in the thylakoid membrane (Vass et al., 1992; Telfer et al., 1994). Photodamaged PsbA is rapidly turned over and replaced by the de novo synthesized copy and this constitutes the repair mechanism that is crucial for the survival of plants exposed to high irradiance. PsbA photodamage, along with a decreases in the quantum yield of photosynthesis and in the light-saturated activity of PSII, is a symptom of photoinhibition, i.e. the decrease in photosynthetic activities encountered by plants exposed to irradiances that exceed the level experienced during their growth history (Šetlik et al., 1990). Two photoinhibition mechanisms have been suggested to operate in vivo: the acceptor-side one, being a consequence of the generation of singlet oxygen after $\mathrm{Phe}^{-} / \mathrm{P} 60^{+}$ charge recombination, and the donor-side one, involving generation of superoxide anion radicals on the donor side of PSII (Andersson \& Aro, 2001).

Degradation of PsbA photodamaged as a result of the acceptor-side photoinhibition events occurs through a two-step process. First, the photodamaged molecule migrates to the stroma-exposed regions of the thylakoid membrane and is cleaved there into N-terminal 23-kDa and C-terminal 10-kDa fragments (Greenberg et al., 1987; Canovas \& Barber, 1993). This primary cleavage occurs at the stromal loop connecting the transmembrane $\alpha$-helices $\mathrm{D}$ and $\mathrm{E}$ and is mediated by the thylakoid membrane-associated Deg2 protease in a GTP-dependent manner (Haussuhl et al., 2001). Then the N-terminal 23-kDa fragment is degraded in an ATP-dependent process by a thylakoid membrane-bound metalloprotease belonging to the FtsH family. It has not been unequivocally determined which individual $\mathrm{FtsH}$ protease(s) is responsible, with FtsH1, 2 and 5 being the strongest candidates (Lindahl et al., 2000; Bailey et al., 2002; Sakamoto et al., 2002). Degradation of PsbA photodamaged as a consequence of donorside photoinhibition requires prior covalent linking of the molecule to other, unidentified PSII proteins (Andersson \& Aro, 2001). The photodamaged molecule is cleaved at the stromal DE-loop and/or lumenal AB-loop to a few degradation products, but the protease(s) involved have not been identified yet (Andersson \& Aro, 2001).

PsbD

PsbD (traditionally designated D2) is a 39.5 $\mathrm{kDa}$, chloroplast genome-encoded protein, homologous to PsbA (Fig. 2). It is integrally associated with the thylakoid membrane, and forms there the PsbA/PsbD heterodimer of the reaction center of PSII which binds the primary chlorophyll donor P680 as well as two peripheral chlorophylls $a$ and one $\beta$-carotene along with all the cofactors mediating the electron flow $\left([\mathrm{Mn}]_{4}, \mathrm{Tyr}_{\mathrm{Z}^{\prime}}\right.$ pheophytin $a$, $\mathrm{Q}_{\mathrm{A}^{\prime}}, \mathrm{Q}_{\mathrm{B}}$ and nonheme Fe). PsbD comprises, as in the case of PsbA, five transmembrane $\alpha$-helices (A to E) and two surface $\alpha$-helices localized between $C$ and D (luminal) and D and E (stromal) ones (Michel \& Deisenhofer, 1988; Barber et al., 1997; Ferreira et al., 2004). The organization of PsbD transmembrane $\alpha$ -

PsbA: MTAILER--RESESLWGRFCNWITSTENRLYIGWFGVLMIPTLLTATSVF

PsbD: MTIALGKFTKDEKDLFDIMDDWLRR-DRFVFVGWSGLLLFPCAYFALGGW

PsbA: IIAFIAAPPVDIDGIREPVSGSLLYGNNIISGAIIPTSAAIGLHFYPIW-

PsbD: FTGTTFVTSWYTHGL----ASSYLEGCNFLTAAVSTPANSLAHSLLLLWG

PsbA: -EA-ASVDEWLYNGGPYELIVLHFLLGVACYMGREWELSFRLGMRPWIAV

PsbD: PEAQGDFTRWCQLGGLWAFVALHGAFALIGFMLRQFELARSVQLRPYNAI

PsbA: AYSAPVAAATAVFLIYPIGQGSFSDGMPLGISGTFNFMIVFQAEHNILMH

PsbD: AFSGPIAVFVSVFLIYPLGQSGWFFAPSFGVAAIFRFILFFQGFHNWTLN

PsbA: PFHMLGVAGVFGGSLFSAMHGSLVTSSLIRETTENESANEGYRFG--QEE PsbD: PFHMM----------CAIHGATVENTLFED---GDGANTERAFNPTQAE

PsbA: ETYNIVAAHGYFGRLIFQYASFNNSRSLHFFLAAWPVVGIWFTALGISTM PsbD: ETYSMVTANRFWSQ-IFGVA-FSNKRWLHFFMLFVPVTGLWMSAQPHENL

PSbA: AFNLNGFNFNQSVVDSQGRVINTWADI INRANLGMEVMHERNAHNFPLDL PsbD: LGVV-GLALNLRAYDFVSQEIRAAEDPEFETFYTKNILLNEGIRAWMAAQ

PsbA: -AAVEAPSTNG

PsbD: DIFPEEVLPRGNAL
Figure 2. Alignment of PsbA and PsbD primary structure in Arabidopsis.

Transmembrane $\alpha$-helices are boxed in grey. Amino acids which are conserved in both PsbA and PsbD are shown in red. Sequences were obtained by BLAST search and alignment of GeneBank Database. 
helices is identical to that of the organization of the $\mathrm{M}$ subunit from photosynthetic purple bacteria reaction center (Deisenhofer et al, 1985; Ferreira et al., 2004).

PsbD binds two chlorophylls out of four constituting P680 - namely $\mathrm{P}_{\mathrm{D} 2}$ (by His197), and $\mathrm{Chl}_{\mathrm{D} 2}$ (the closest residue is Ile178), and the peripheral chlorophyll a molecule $\mathrm{Chl}_{\mathrm{ZD} 2}$ (Ferreira et al., 2004).

His197 of PsbD is the second ligand for P680, besides His197 of PsbA. PsbD Phe261 and His214 are, in turn, involved in $\mathrm{Q}_{\mathrm{A}}$ binding (domain $\mathrm{A}$ ) and His214 is a ligand of nonheme Fe (Ferreira et al., 2004).

One $\beta$-carotene molecule assigned to the $\mathrm{PsbA} / \mathrm{PsbD}$ heterodimer may be functionally related to PsbD - this pigment molecule is suggested to transfer the excitation energy from $\mathrm{Chl}_{\mathrm{ZD} 2}$ to $\mathrm{P} 680$ (Ferreira et al., 2004). The same $\beta$-carotene is thought to mediate electron flow between PsbE/F (the low molecular mass protein of PSII reaction center) and P680 as an element of the secondary electron transfer pathway within PSII, helping to protect it against photodamage (Stewart \& Brudvig, 1998).

PsbD (as well as PsbA) are reversibly phosphorylated by redox-regulated kinases but the functional meaning of this process has not been explained yet (Callahan et al., 1990; Koivuniemi et al., 1995).

Using deletion mutagenesis it has been shown that the C-terminal domain of PsbD is important for the function and stability of PSII. Downregulation of PsbD resulted in the loss of the ability to photoautotrophic growth and the decrease in the number of functional PSII reaction centers in thylakoid membranes (Eggers \& Vermaas, 1993).

PsbB

PsbB is a pigment-binding protein of PSII of a molecular mass of about $56 \mathrm{kDa}$, encoded by a single gene localized in the chloroplast genome. This protein is integrally associated with the thylakoid membrane and constitutes a polypeptide moiety of $\mathrm{CP} 47$, one of two inner light-harvesting complexes of PSII (the second one is CP43) (Barber et al., 1997; Bricker \& Frankel, 2002).

Data obtained from electron microscopy (Rhee et al., 1998) and X-ray crystallography (Zouni et al., 2001) demonstrated that PsbB traverses the thylakoid with six $\alpha$-helices (designated I-VI) which are separated by five extrinsic loop domains (A-E). The $\mathrm{N}$ - and C-termini of the molecule as well as the $\mathrm{B}$ and $\mathrm{D}$ loops are exposed to the stroma while the A, C and E loops are buried in the thylakoid lumen (the E loop is specially large and comprises 193 residues). The structure of the transmembrane $\alpha$-helices is well recognized on the maps of PsbB whereas the structure of the hydrophilic loops - involving a re- markable number of PsbB amino-acid residues is not visible on the map of PsbB structure.

It has been established that single PsbB molecule binds 16 chlorophylls $a$ and 2 to $3 \beta$-carotenes (Ferreira et al., 2004). Most of the chlorophylls, except for one, are arranged in two layers on opposite sides of the membrane. Thirteen chlorophyll $a$ molecules are ligated by histidine residues, located within predicted membrane-spanning regions (Barber et al., 1997). The association of two other chlorophyll molecules is most probably facilitated by methionine or serine side chains whereas one chlorophyll has not been assigned to any ligand yet (Ferreira et al., 2004).

CP47 (and CP43) are associated with the PbsA/PsbD heterodimer, with CP47 located closer to PsbD and CP43 closer to PsbA. There are suggestions that CP47 is located in the middle of the dimeric PSII core complex, near the interface of the two monomers, since the so called CP47-reaction centers may be isolated, containing CP47 as well PsbA/PsbD heterodimer, PsbE/F and some other low molecular mass proteins, but not CP43 (Akabori et al., 1988).

CP47 acts mainly as an inner light harvesting complex transferring excitation energy from peripheral light-harvesting complexes to the PsbA/PsbD heterodimer. Chlorophyll a molecules bound to the polypeptide moiety of the complex absorb light in the red spectral region. Three groups of pigments with absorption bands peaking at 690 and about 680 and $670 \mathrm{~nm}$ have been identified using various spectroscopic approaches (Groot et al., 1995). The variability of the strength of excitonic coupling of individual chlorophylls of CP47 suggests that both the Dexter and Förster mechanisms participate in the flow of excitation energy within the complex (Groot et al., 1995). The $\beta$-carotenes associated with $\mathrm{PsbB}$ are in close contact with chlorophylls and are engaged in a singlet-singlet $\beta$-carotene/chlorophyll $a$ excitation energy transfer (Ferreira et al., 2004). In addition, CP47 (and CP43) appears to interact with three PSII extrinsic proteins (PsbO, P and Q), which function as enhancers of the oxygen evolution process. Genetic and biochemical evidence indicates that CP47 - together with CP43 - may help form the binding site for these extrinsic proteins to the remaining part of PSII (Bricker \& Frankel, 1988).

Deletion of the $P s b B$ gene and site-directed mutational studies indicated that CP47 is very important for PSII assembly and functions (Meurer et al., 1996).

$\mathrm{PsbC}$

PsbC is a thylakoid-membrane-associated, pigment-binding protein representing the polypeptide moiety of CP43, one of two inner PSII light harvesting complexes. PsbC is encoded by a single 
chloroplast gene and has a molecular mass of about $50 \mathrm{kDa}$, depending on species (Barber et al., 1997; Bricker \& Frankel, 2002).

PsbC is homologous to PsbB (Fig. 3) and precisely following what has been established for PsbB - spans the thylakoid membrane with six $\alpha$ helices (I-VI) with both amino and carboxyl termini as well as the $\mathrm{B}$ and $\mathrm{D}$ loops exposed to the stromal surface and the A, C and E loops (E one is specially large) exposed to the luminal surface (Barber et al., 1997; Bricker \& Frankel, 2002).

A single PsbC molecule has been demonstrated to be associated with 14 chlorophylls and 3-4 $\beta$-carotenes. Ten of the chlorophylls are ligated by histidine residues and one by asparagine (Ferreira et al., 2004). As in the case of CP47 most of the chlorophylls belonging to CP43 - except for one are arranged in two layers on opposite sides of the membrane. X-Ray crystallography data suggest that CP43 occupies a more peripheral postion within PSII core complex than CP47 (Rhee et al., 1998; Zouni et al., 2001). It is in agreement with the observations showing that PsbC protein is more weakly associated with the PSII reaction center than PsbB and is much easier to remove from PSII than CP47 (Akabori et al., 1988).

CP43 acts mainly as an inner light-harvesting complex cooperating with $\mathrm{CP} 47$ in transferring the excitation energy from peripheral light-harvesting complexes to the PsbA/PsbD heterodimer. Chlorophyll $a$ molecules bound to the polypeptide moiety of the complex absorb light in red spectral region and have spectroscopic features very similar to those of CP47 except that CP43 has a sharp absorption band at $682.5 \mathrm{~nm}$ instead of the $690 \mathrm{~nm}$ band found in CP47. Both the Dexter and Förster mechanisms seemingly participate in the flow of excitation energy within the complex (Groot et al., 1995; 1999). A few out of the seven $\beta$-carotenes assigned to $\mathrm{CP} 43$ are observed in the $\mathrm{CP} 43 / \mathrm{PsbA}$ interface and thus may directly mediate the exctitation energy transfer between the inner light-harvesting complexes and the PsbA/PsbD heterodimer (Ferreira et al., 2004).

There is some evidence that removing CP43 has an impact on photoreduction of $\mathrm{Q}_{\mathrm{A}}$. This may imply that PsbC plays a role in the stabilization of $\mathrm{Q}_{\mathrm{A}}$ binding by PsbD. Moreover, the inability to accumulate PsbC leads to reduced level of PsbA, PsbB and PsbD. These observations were interpreted as suggesting that PsbC stabilizes other large pigmentbinding proteins of PSII core complex but the role of PsbC in stabilizing $Q_{A}$ PsbA, PsbB and PsbD is a matter of controversy (Petersen et al., 1990; Rogner et al., 1991). In addition, CP43 appears, as mentioned above, to cooperate with $\mathrm{CP} 47$ in interacting with three PSII extrinsic proteins (PsbO, P and Q), which function as enhancers of oxygen evolution process (Bricker \& Frankel, 1988).

PsbC occurs in vivo as a population of two unphosphorylated forms and a form which is phosphorylated at its N-terminal threonine residue (Andreucci et al., 2005). The functional meaning of this heterogeneity is not clear.

The results of studies with mutants devoid of PsbC suggest that it is necessary for PSII to be assembled and function properly (Rogner et al., 1991).

PsbS

PsbS is a $22 \mathrm{kDa}$ protein encoded by a single, nuclear gene, integrally associated with the thylakoid membrane (Kim et al., 1992; Jansson, 1999). In spite of traversing the membrane with four transmembrane $\alpha$-helices (designated I-IV), PsbS displays a strong homology with the Lhcb type apoproteins (Fig. 4), unequivocally predicted to have three transmembrane $\alpha$-helices (designated A-C, see above paragraph PsbD). The largest extent of homology is
PsbB: GLPWYRVHTVVLNDPGRLLAVHIMHTALVAGWAGSMALYELAVFDPSDPVLDPMWRQGMF PsbC: GFAWWAGNARLINLSGKLLGAHVAHAGLIVFWAGAMNLFEVAHFVPEKP----MYEQGLI

PsbB: KYARRAQLGEIFELDRATLKSDGVFRSSPRGWFTFGHASFALLFFFGH IWHGARTLFRDV PsbC: EYMTHAPLGSLNSVGGVATEINAVNYVS PRSWLSTSHFVLGFFLFVGHLWHAGRA--RAA

PsbB: IFGIHLFLSGVACF--GFGAFHVTGLY---GPGIWVSDPYGLTG-KVQP-------VNPA PsbC: ILGIHLILLGVGAFLLVFKALYFGGVYDTWAPG--GGDVRKITNLTLSPSVIFGYLLKSP

PsbB: WGVEGFDPFVPG--GIASHHIAAGTLGILAGLFHLSVRPPQRLYKGLRMGNIETVLSSSI PsbC: FGGEGWIVSVDDLEDI I GGHVWLGS ICIFGGIWHI LTKPFAWARRALVWSG-EAYLSYSL

PsbB: --_-------GTMWYGSATTP IELFGPTEGVAGAHIVFSGLCFLAAIWHDLPKIFGIHL PsbC: AALSVCGFIACCFVWFNNTAYPSEFYGPTEDI I GGHVWLGS ICIFGGIWHDLEDI I GGHV

PsbB: FLSGVACFGFGAFHVTGLYGPGIWKIFGIHLFLSGVACFGFGAFHVTGLYGPGIWVSD-P PsbC: WLGSICIFG-GIWHI--LTKPFAWKLLGAHVAHAGLIVFWAGAMN---LFEVAHFVPEKP

PsbB: -Y--GLTGKVQPVNPAWGV----E GFD-PFVPGGIASHHIAAGTLGILAGLFHLSVRP PsbC: MYEQGLILLPHLATLGWGVGPGGE-VIDTFPYFVSGVL-HLISSAVLGF-GGIYHALLGP

PsbB: GHASFALLFFFGHIWHGHIWHGARTLFRDVF

PsbC: GHVWLGSICIFGGIWHGHVWLGSICIFGGIW
Figure 3. Aligment of PsbB and PsbC primary structure in Arabidopsis.

Transmembrane $\alpha$-helices are boxed in grey. Amino acids which are conserved in both PsbB and $\mathrm{PsbC}$ are shown in red. Sequences were obtained by BLAST search and alignment of GeneBank Database. 


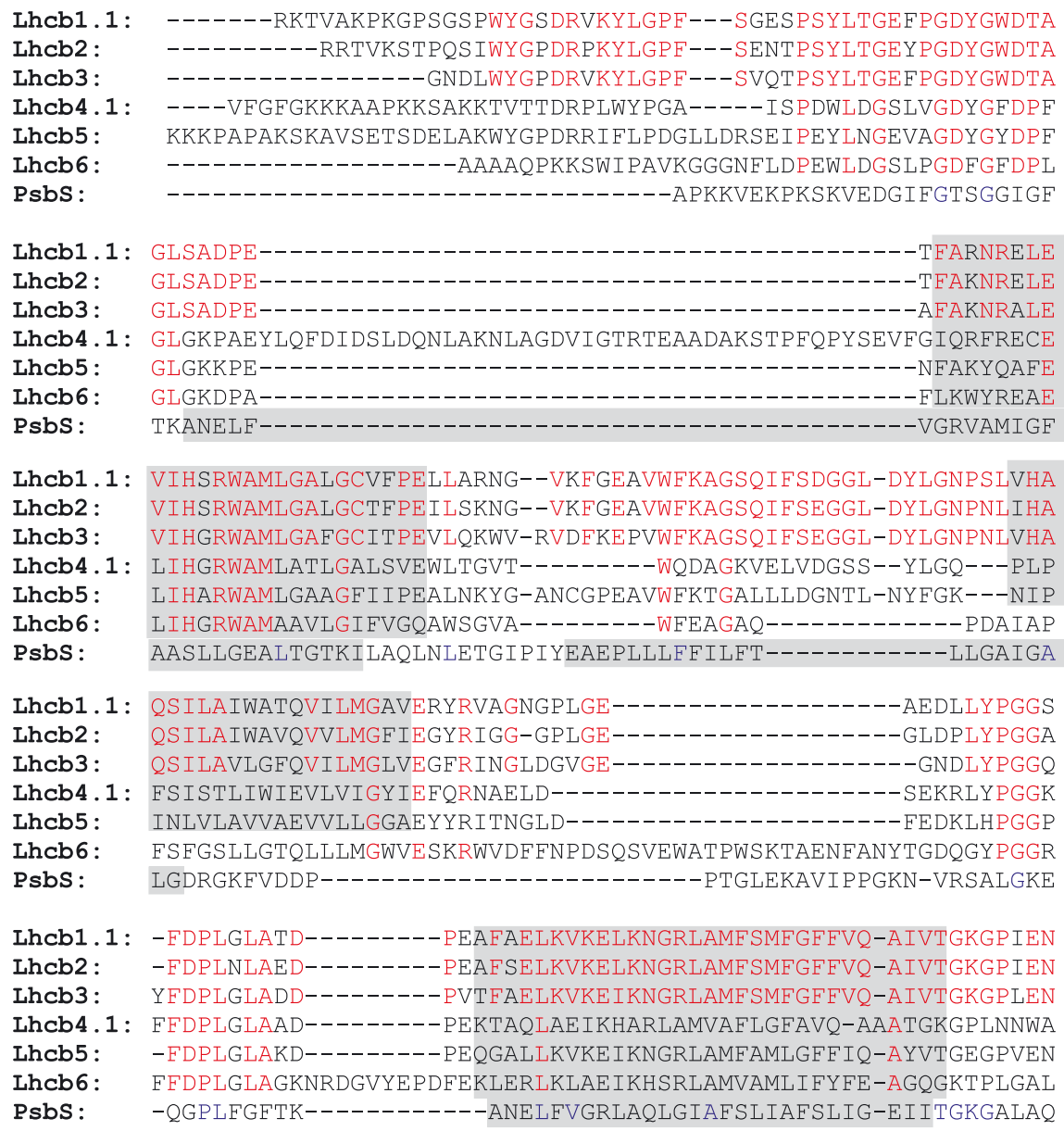

Lhcb1. 1: LADHLADPVNNNAWAFATNFVPGK

Lhcb2: LFDHLADPVANNAWSYATNEVPGK

Lhcb3: LLDHLDNPVANNAWAFATKFAPGA

Lhcb4.1: THLSDPLHTTIIDTFSSS

Lhcb5: LAKHLSDPFGNNLLTVIAGTAERAPTL

Lhcb6: GL

Psbs: LNIETGIPIQDIEPLVLLNVAFFFFAAINPGNGKFITDDGEES

Figure 4. Aligment of Lhcb1-6 and PsbS primary structure in Arabidopsis.

Transmembrane $\alpha$-helices are boxed in grey. Amino acids which are conserved in Lhcb1-6 or in Lhcb1-3 are shown in red. Those amino acids of which are conserved in Lhcb1-3 and PsbS are labelled in blue in PsbS sequence. Sequences were obtained by BLAST search and alignment of GeneBank Database.

found in PsbS $\alpha$-helices I and III and the A/B $\alpha$-helices of Lhcb apoproteins (Kim et al., 1992).

PsbS has been shown to bind chlorophyll $a / b$ and xanthophylls (Funk et al., 1995) but this notion has recently been severely questioned by the results of studies which demonstrated that neither purified nor recombinant PsbS binds chlorophyll and that PsbS does not refold in vitro with pigments (Dominici et al., 2002). Following in vitro refolding the recombinant protein assumes, however, a conformation similar to that observed in vivo (without pigment binding) and it is concluded that PsbS may in fact bind pigments in situ but in a manner different from what was described for any other PSII pigment-binding protein (Dominici et al., 2002). This assumption is the reason why PsbS is included in this review, devoted to pigment-binding proteins of PSII.

There is a vigorous debate with respect to the localization of PsbS within the PSII particle (Hankamer et al., 1997; Dominici et al., 2002). Recent findings suggest that PsbS is either associated with PSII core complex or lies between the core and the CP29/CP26 peripheral light harvesting complexes (Dominici et al., 2002).

Ample experimental evidence has been accumulated in favour of the notion that PsbS plays a very important role in photoprotection by being specifically involved in the development of the $\mathrm{qE}$ component of the non-photochemical quenching (NPQ) phenomenon ( $\mathrm{Li}$ et al., 2000; 2002). It is suggested that the decrease in the thylakoid lumen $\mathrm{pH}$ 
value occurring under excessive light conditions leads to the protonation of two PsbS glutamate residues (Glu122 and Glu126) and to zeaxanthin binding to PsbS. The protonated PsbS-zeaxanthin complex is thought to impose conformational changes on the neighbouring light-harvesting complexes that effectively switches the PSII unit into a quenched state in which non-photochemical de-excitation of $\mathrm{Chl}^{*}$ is favoured (Aspinal O'Dea et al., 2002; Li et al., 2004).

\section{PIGMENT-BINDING PROTEINS OF PSII PERIPHERAL LIGHT-HARVESTING COMPLEXES}

Peripheral light-harvesting complexes of PSII of higher plants and green algae are composed of pigments and lipids associated with six types of apoproteins, Lhcb 1-6. Lhcb1 along with Lhcb2 and Lhcb3-type apoproteins together consitute the polypeptide moiety of LHCII - by far the most abundant peripheral energetic antenna complex of PSII, accounting for about $60 \%$ of the total chlorophyll content of thylakoid membranes. Lhcb4, Lhcb5 and Lhcb6 consitute, in turn, the polypeptide moiety of minor peripheral light-harvesting complexes, namely CP29, CP26 and CP24, respectively.

\section{Structure of proteins of PSII peripheral light-har- vesting complexes}

\section{Lhcb1}

Apoproteins of the Lhcb1 type are usually encoded by several nuclear Lhcb1 genes (Jansson, 1999) but the number of copies may be as high as 16 (Dunsmuir, 1985). Individual $L h c b 1$ genes encode apoproteins differing slightly with regard to the apparent molecular mass (24.8-25.0 kDa range as determined by RP-HPLC-ESI-MS; Zolla et al., 2003), and some apoproteins may be represented by isoforms having various pI values (Jackowski \& Przymusiński, 1995). The amino-acid composition of Lhcb1 is highly dominated by hydrophobic residues; glycine, alanine and leucine may account for more than $35 \%$ of residues (Jansson, 1994).

The structure of pea and spinach LHCII has been determined by X-ray crystallography and includes all amino-acids except for 9-14 N-terminal ones (they are not visible in the structure) as well as all chlorophyll, carotenoid and lipid molecules (Liu et al., 2004; Standfuss et al., 2005). As Lhcb1 and Lhcb2 together account for $89 \%$ of the apoprotein content of LHCII (Jackowski \& Jansson, 1998; Jackowski et al., 2001) and the primary structure of pea Lhcb1 and Lhcb2 is virtually identical with the exception for several $\mathrm{N}$-terminal residues (Standfuss \&
Kühlbrandt, 2004), the X-ray structure of LHCII represents in fact that of Lhcb1 as well as Lhcb2. The lack of visibility of the N-terminal residues on the X-ray map of LHCII structure may be ascribed to the simultaneous presence of two types of N-terminal sequences in the LHCII crystals analysed.

Lhcb1 is integrally associated with the thylakoid membrane and spans the membrane with three $\alpha$-helices labelled B, C and A, with the $\mathrm{N}$-terminus of the molecule facing the stroma and the $\mathrm{C}$-terminus penetrating the lumen. The $\alpha$-helices B and $\mathrm{A}$ are tilted by $32^{\circ}$ with regard to the membrane normal plane and form an X-like structure kept by an attractive force between the charged residues of Arg70(B) with Glu180(A) and Glu65(B) with Arg185(B). These helix-helix interactions are thought to play an important role in stabilizing Lhcb1 in the thylakoid membrane (Kühlbrandt et al., 1994). The $\alpha$-helix C is considerably shorter than the $\mathrm{A}$ and $\mathrm{B}$ ones and has a tilt angle of $9^{\circ}$. Besides transmembrane helices B, $C$ and $A$, the Lhcb1 structure comprises two short, lumen-exposed amphipatic $\alpha$-helices, referred to as D and E (Kühlbrandt et al., 1994).

An individual Lhcb1 molecule is assumed to bind eight chlorophyll $a$, six chlorophyll $b$, four carotenoid and two lipid molecules (Liu et al., 2004; Standfuss et al., 2005). In contrast to the data coming from mutational analysis - pointing to a mixed occupancy by chlorophyll $a$ and chlorophyll $b$ molecules of three chlorophyll-binding sites (Remelli et al., 1999) - X-ray crystallographic analysis demonstrates that all chlorophyll-binding sites in the Lhcb1 molecule are occupied by one type of chlorophyll. The ligands of 14 chlorophylls have been determined to be: seven amino-acid residues, two backbone carbonyls, four water and one phosphatidylglycerol molecule (Liu et al., 2004). The chlorophyll molecules are distributed in two layers, one (eight molecules) lying at the stromal surface of Lhcb1 and the second (remaining six molecules) at the lumenal surface. Carotenoid molecules are associated with Lhcb1 at four sites, called L1, L2, N1 and V1, differing with regard to binding strength. Sites L1 and L2, cross-bracing the A and B helices, are occupied by tightly bound lutein (L1) and lutein (90\%) and violaxanthin (10\%) (L2), whereas site N1, located in the region around helix $\mathrm{C}$, tightly binds neoxanthin (Croce et al., 1999; Liu et al., 2004). The fourth site, called V1, binds violaxanthin and other xantophyllcycle carotenoid molecules in a loose manner, so that they are lost during LHCII purification by nondenaturing isoelectrofocusing (Caffari et al., 2001a). $\mathrm{V} 1$ is located in the interface between two adjacent Lhcb1 molecules (Liu et al., 2004). In addition to photosynthetic pigments Lhcb1 binds different lipids, most probably one phosphatidylglycerol molecule (at the interface between two Lhcb1s) and three 
digalactosyl diacylglycerol ones (on the lumenal side of Lhcb1) (Standfuss et al., 2005).

LHCII within the thylakoid membrane is a population of trimeric pigment-protein assemblies differing with regard to their apoproteins' composition. The Lhcb1 type apoprotein seems to be indispensable for LHCII trimer formation in vivo since only Lhcb1containing assemblies (namely Lhcb1 ${ }_{3}$, Lhcb1 2 Lhcb2, Lhcb1 $1_{2}$ Lhcb3 and Lhcb1 Lhcb2 Lhcb3) have been purified from thylakoid membranes (Jackowski \& Jansson, 1998; Jackowski et al., 2001). In fact, LHCII trimers containing only Lhcb2 or Lhcb2 and Lhcb3 types can be formed in vitro by refolding Lhcb2 and Lhcb3 with purified pigments (Standfuss \& Kühlbrandt, 2004) but these kinds of trimers have never been observed in vivo. It has been established that the Lhcb1 region engaged in trimerization comprises the $\mathrm{N}$ - and C-terminal domains, the stromal end of $\alpha$-helix B, some amino-acid residues of $\alpha$-helix $\mathrm{C}$, as well as pigment and lipid molecules bound to these regions of Lhcb1 (Hobe et al., 1995; Kuttkat et al., 1996; Liu et al., 2004).

Lhcb2

The apoproteins of Lhcb2 type are encoded, as in the case of Lhcb1 apoprotein, by several nuclear Lhcb2 genes (Jansson, 1999). The molecular mass of individual apoproteins of Lhcb2 type measured by RP-HPLC-MS-ESI falls within the 24.6-24.9 $\mathrm{kDa}$ range (Zolla et al., 2003). Hydrophobic residues highly dominate the amino-acid composition of Lhcb2-type apoproteins as in the case of Lhcb1 (Jansson, 1994). The Lhcb2 and Lhcb1 types of apoproteins are very similar to each other so that their primary structures yield up to $94 \%$ identity depending on species (Fig. 4) (Jansson, 1994; Standfuss \& Kühlbrandt, 2004).

Lhcb2 type apoproteins account for $11-33 \%$ of total LHCII apoprotein content and thus add appropriately to the structure of the LHCII holocomplex revealed by X-ray crystallography - which thus reflects the features of both Lhcb1 and Lhcb2 types of apoproteins. Although not all chlorophyll-binding amino acid resides are conserved in Lhcb1 and Lhcb2, the composition and arrangement of chlorophylls bound to Lhcb1 and Lhcb2 (as well as that of carotenoids and lipids) is thought to be virtually identical (Liu et al., 2004; Standfuss et al., 2005).

Lhcb2 occurs in vivo exclusively as heterotrimers with Lhcb1 (Jackowski \& Jansson, 1998) or Lhcb1 and Lhcb3 (Jackowski et al., 2001) in spite of the fact that it may readily form homotrimers and heterotimers with Lhcb3 in in vitro reconstitution experiments (Standfuss \& Kühlbrandt, 2004).

Lhcb3

The apoproteins of the Lhcb3 type are coded by one-four Lhcb3 type nuclear gene (Jansson, 1994;
1999). The proteins lack the N-terminal domain present in both Lhcb1 and Lhcb2, and that is the reason why Lhcb3 is $5-10$ residues shorter than the products of the $L h c b 1$ and $L h c b 2$ genes. The remaining fragment of the Lhcb3 molecule is more divergent from both Lhcb1 and Lhcb2 than they are from each other - in Arabidopsis the Lhcb3 primary structure is $74 \%$ identical with that of Lhcb1 and $80 \%$ with that of Lhcb2 (Fig. 4) (Jansson, 1999). The molecular mass of Lhcb3 measured by RP-HPLC-MSESI equals 24.2-24.4 kDa (Zolla et al., 2003).

The Lhcb3 type apoprotein never accounts for more than $11 \%$ of the apoprotein content of LHCII in vivo (Jackowski \& Jansson, 1998; Jackowski et al., 2001) and thus adds weakly to LHCII overall structure. The topology of Lhcb3 within the thylakoid membrane is most probably identical with that of Lhcb1 and Lhcb2 but the composition and arrangement of the array of pigments bound to Lhcb3 may be slightly divergent from what was established for Lhcb1 and Lhcb2. HPLC-based determinations suggest that Lhcb3 may bind only 13 chlorophyll molecules (one chlorophyll $b$ lacking) and that the N1 site in some Lhcb3 molecules may be empty (Caffari et al., 2001b; Standfuss \& Kühlbrandt, 2004). The V1 site is most probably not present at all (Morosinotto et al., 2003).

Lhcb3 occurs in vivo as a heterotrimer with Lhcb1 (Jackowski \& Jansson, 1998) or Lhcb1 and Lhcb2 (Jackowski et al., 2001). Lhcb3 homotrimers were neither observed in vivo nor reconstituted in vitro, although the sequence of all the domains engaged in Lhcb1 trimerization is conserved in Lhcb3 as well (Standfuss \& Kühlbrandt, 2004).

Lhcb4

The apoprotein of Lhcb4 type is coded for by 1-3 nuclear genes (Jansson, 1999). The molecular mass of individual apoproteins measured by RPHPLC-ESI-MS falls within the 28.1-28.8 kDa range (Zolla et al., 2003). The amino-acid composition of Lhcb4 is highly similar to that of Lhcb1-3 (as well as Lhcb5 and Lhcb6) (Fig. 4) but in spite of this it has a low level of sequence identity (30\%) with regard to those apoproteins. The main reason for this is the occurrence of a relatively long (about 40 residues) insertion, localized close to the $\mathrm{N}$-terminus of the molecule of Lhcb4 type apoprotein, lacking in all other Lhcbs.

The Lhcb4 type apoprotein constitutes the polypeptide moiety of CP29, one of the minor peripheral light-harvesting complexes of PSII which accounts for 3-8\% of the chlorophyll content of thylakoid membranes (Green, 1982). A crystal structure of CP29 is not available but based on homology between Lhcb4 and Lhcb1-3 type apoproteins it may be implied that the overall topology of Lhcb4 within 
the thylakoid membrane is the same as in the case of Lhcb1-3. A single molecule of apoprotein of Lhcb4 type binds 6 chlorophylls $a$ and 2 chlorophylls $b$. Only two carotenoid-binding sites have been identified in the structure of Lhcb4, i.e. L1 and L2 - they are occupied by lutein (L1) and neoxanthin (50\%) and violaxanthin (50\%) (L2) (Bassi et al., 1993; Morosinotto et al., 2003).

Lhcb4 type apoproteins occur in vivo as a monomer (Sandona et al., 1998).

Lhcb5

The apoprotein of Lhcb5 type is as a rule coded for by a single Lhcb5 type nuclear gene (Jansson, 1999). The molecular mass of individual Lhcb5 type apoproteins has been determined by RP-HPLCESI-MS to span the 26.5-27.9 kDa range (Zolla et al., 2003). Lhcb5 displays the strongest level of sequence identity with regard to Lhcb1 and Lhcb2 (40\%) of all apoproteins of the minor light-harvesting complexes of PSII (Jansson, 1999).

Lhcb5 constitutes the polypeptide moiety of CP26 which accounts for about 3\% of the thylakoid membrane total chlorophyll content (Dainese et al., 1990). Although there is only one Lhcb5 type gene in all species investigated so far, CP26 preprations often yield two polypeptides (Allen \& Staehelin, 1992; Zolla et al., 2003). This may be due to posttranslational processing of a single gene product.

Crystals of CP26 are not available, but, for the reasons explained above, the overall topology of Lhcb5 within the thylakoid membrane is thought to be identical with that of Lhcb1-3. Six chlorophylls $a$, 3 chlorophylls $b$, one lutein, 0.5 violaxanthin and 0.5 neoxanthin have been found associated with a single molecule of Lhcb5 type apoprotein. Lutein occupies the L1 site whereas violaxanthin and neoxanthin occupy the L2 site in a mixed way (Bassi et al., 1993; Morosinotto et al., 2003).

Lhcb5 type apoproteins are represented in vivo by a monomer form (Sandona et al., 1998). Still, in an Arabidopsis mutants in which Lhcb1 and Lhcb2 had been silenced by introduction of a relevant antisense construct, Lhcb5 type apoproteins were identified to be assembled as homotrimers or Lhcb3/Lhcb5 heterotrimers to compensate for the lack of trimers containing Lhcb1 and Lhcb2. The ability of Lhcb5 to assemble as trimers may be due to the fact that a hexapeptide motif localized at the $\mathrm{N}$-terminus, found to be necessary for Lhcb1-3 trimerization, is conserved in Lhcb5 (Hobe et al., 1995; Ruban et al., 2003).

Lhcb6

The apoprotein of Lhcb6 type is encoded by 1-2 nuclear genes (Jansson, 1999). Lhcb6 type apoproteins are the smallest ones of all belonging to
PSII peripheral energetic antennae - their molecular mass has been determined by RP-HPLC-ESI-MS to span the 22.6-22.9 kDa range (Zolla et al., 2003). Furthermore, Lhcb6 is the most divergent from Lhcb1-3 of all the apoproteins of minor light-harvesting complexes of PSII (Jansson, 1999).

Lhcb6 is the polypeptide moiety of CP24 accounting for less than $3 \%$ of the chlorophyll content of the thylakoid membrane (Dainese et al., 1990). CP24 preparations of some species yield more Lhcb6 type apoproteins than the number of Lhcb6 type genes identified, thus the products of the genes must undergo posttranslational modifications changing their molecular masses (Jansson, 1994; Zolla et al., 2003).

CP24 has not been crystallized yet. As the level of sequence identity between Lhcb6 and Lhcb1-3 is low it has been suggested that Lhcb6 might not present the same overall topology within the thylakoid membrane as Lhcb1-3, namely Lhcb6 might not contain regions homologous to $\alpha$-helices $C$ and D of Lhcb1-3 (Green et al., 1991; Cai et al., 1993).

Lhcb6 type apoproteins are represented in vivo by a monomer form (Sandona et al., 1998) and a single molecule of Lhcb6 binds 5 chlorophylls $a, 4$ chlorophylls $b$, one lutein (L1 site) and one violaxanthin (L2 site) (Bassi et al., 1993; Morosinotto et al., 2003).

\section{PSII-LHCII supercomplexes}

When stacked thylakoid membranes are treated with detergents in a mild manner and then subjected to ultracentrifugation, so called PSII-LHCII supercomplexes can be isolated, comprising a dimeric PSII core complex associated with various amounts of peripheral light-harvesting complexes (Fig. 5). Single particle image analysis of electron micro-

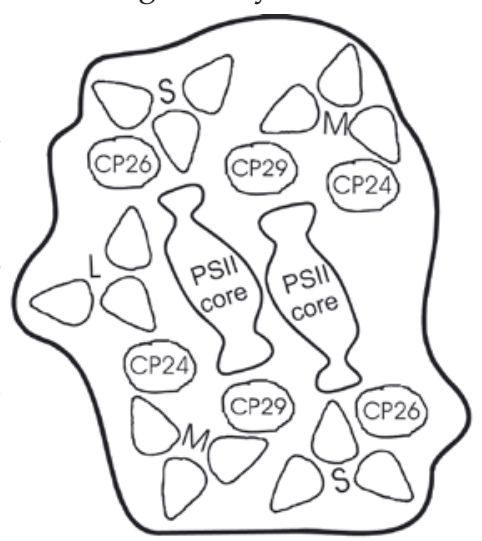

Figure 5. Schematic representation of supramolecular organization of PSII-LHCII supercomplex comprising dimeric PSII core complex, LHCII trimers bound at S, $M$ and $L$ sites and monomeric, peripheral PSII light-harvesting complexes (CP24, CP26, CP29).

Details are not to scale. After Boekema et al. (1999), modified. 
graphs of vitrified samples of solubilized stacked thylakoid membranes allowed to recognize the supercomplexes as having an exceptional, rectangular shape (Boekema et al., 1995). The PSII-LHCII supercoplexes most probably represent the organization of PSII that occurs in appressed thylakoid membranes in vivo since they are found also among products of solubilization of complete thylakoid membranes (Eshagi et al., 1999).

The dimeric PSII core complex binds LHCII trimers at three types of sites, differing with regard to binding strength, designated $\mathrm{S}, \mathrm{M}$ and $\mathrm{L}$ (strong, medium and loose association, respectively). Two individual binding sites of each type have been found to be present in the supercomplexes so that the dimeric core may bind six LHCII trimers at the most. It has been established that the supercomplexes isolated from various species have various numbers of the binding sites occupied with LHCII trimers, e.g. Arabidopsis ones contained M and S-type trimers while in spinach a certain (very tiny) fraction of supercomplexes had the trimers associated only at the S and L sites (Boekema et al., 1999; Yakushevska et al., 2001). Anyhow, no more than four trimers have been found to be bound to the dimeric PSII core complex, including data coming from studies performed with algae (Nield et al., 2000) and liverworts (Harrer, 2003). It has been suggested that S-type LHCII trimers preferentially contain Lhcb1 and Lhcb2 gene products while M-type ones contain Lhcb3 apoproteins as well (Hankamer et al., 1997; Boekema et al., 1999). M-type trimers are connected to the PSII core complex directly or through S-type ones, depending on species, L-ones are thought to be in direct contact with the core (Boekema et al., 1999; Yakushevska et al., 2001).

The minor peripheral energetic antennae of PSII are thought to be represented by two copies per dimeric PSII core complex. Two CP26 particles are most probably localized at the tips of the rectangular PSII-LHCII supercomplex, near CP43 (Yakushevska et al., 2003), whereas CP29 is thought to be in close vicinity of $\mathrm{CP} 47$ and serve as an attaching point for S-type LHCII trimers (Boekema et al., 1999; Yakushevska et al., 2003). The location of CP24 has not been identified unequivocally, it was established, however, that it occurs exclusively in the supercomplexes containing both S- and M-type trimers, near CP29 (Hankamer et al., 1997; Boekema et al., 1999).

In Arabidopsis mutants in which Lhcb1 and Lhcb2 are silenced the relevant apoproteins bound at the $\mathrm{M}$ and $\mathrm{S}$ sites are replaced by Lhcb5 and Lhcb3 whose expression in the mutants is strongly upregulated. The structural organization of PSIILHCII supercomplexes with LHCII replaced by Lhcb3 and Lhcb5 in a compensatory way is virtually identical to the ones of wild-type plants (Ru- ban et al., 2003). The effect of replacement is most probably unique to the Lhcb1 and Lhcb2 type apoproteins - mutants lacking Lhcb5 have PSII-LHCII supercomplexes with the CP26 site empty while in mutants lacking Lhcb4 the supercomplexes are virtually absent. This demonstrates the seminal role of CP29 in the stability of the supercomplexes (Yakushevska et al., 2003).

The results of biochemical studies suggest that in native stacked thylakoid membranes the stoichiometric ratio between LHCII trimers and the dimeric PSII core complex is 8:1 (Morrisey et al., 1988; Dainese et al., 1990). A possible explanation of the discrepancy between this figure and the findings that seemingly no PSII-LHCII supercomplexes bind more than four LHCII trimers (Boekema et al., 1999) is that some aggregated trimers have been found that do not remain associated with PSII core but diffuse freely in the membrane instead (Jackowski \& Kluck, 1994; Dekker et al., 1999).

No PSII-LHCII supercomplexes have been found to occur in non-stacked thylakoid membranes. PSII residing in these domains are recovered as monomeric cores with no or very limited array of peripheral light-harvesting complexes attached to them (Jansson et al., 1997; Dekker et al., 2002).

Image analysis of electron micrographs of stacked thylakoid membranes shows that PSII-LHCII supercomplexes can associate laterally in specific ways (in rows) to form a few types of so called megacomplexes. The M-type sites and CP29 are required for the formation of the majority of megacomplexes (Boekema et al., 1999). It is not certain whether the PSII megacomplexes reflect the native arrangement of PSII particles in situ.

\section{Functions of proteins of PSII peripheral light-har- vesting complexes}

The main function of LHCII is to harvest solar energy and deliver it, in the form of excitation energy, to PSII core complex. Chlorophyll $a$ and $b$ molecules bound to the polypeptide moiety of the complex absorb light in the red spectral region. The variability of microenvironment of individual chlorophylls determined by the surrounding polypeptide matrix is the reason why the LHCII absorption spectrum displays several spectral bands, covering the 630-685 nm range (Hemelrijk et al., 1992; Rogl et al., 2002). The pairs of chlorophylls which are the closest neighbours within an LHCII monomer have an excitonic character and exchange energy according to the Dexter mechanism (chlorophyll $b /$ chlorophyll $a$, chlorophyll $a /$ chlorophylla $a$ and chlorophyll $b /$ chlorophyll $b$ Q $\mathrm{Q}_{\mathrm{y}}-\mathrm{Q}_{\mathrm{y}}$ singlet-singlet transfers are allowed) on a time scale of less than 6 ps (Gradinaru et al., 1998). The exctitation energy moves from the 
most blue-shifted chlorophyll (not unequivocally identified yet) to the most red-shifted one, suggested to be a chlorophyll $a$ molecule, labelled Chl 2 (Liu et al., 2004; Standfuss et al., 2005), which plays the role of the terminal fluorescence emitter within the LHCII monomer. This chlorophyll then passes the excitation energy according to the Förster mechanism to the pigments of neighbouring LHCII monomers, minor light-harvesting complexes and, finally P680 (Standfuss et al., 2005). Lutein and neoxanthin molecules of LHCII are engaged in harvesting solar energy as well due to absorbing light in the bluegreen spectral region. The pathways of excitation energy transfer involving carotenoids are much less explored than the ones solely involving chlorophylls, nevertheless, it has been established that a remarkable part of the carotenoid-chlorophyll excitation energy movement occurs according to the Dexter mechanism, due to $S_{2}-Q_{x}$ singlet-singlet transfers. The time scale is about $100 \mathrm{fs}$. Lutein transfers the energy exclusively toward chlorophyll $a$ while neoxanthin mostly to chlorophyll $b$ (Gradinaru et al., 2000). It is thought that the mechanism and timescale of excitation energy transfer among the pigment molecules of minor light-harvesting complexes of PSII is much the same as in the case of LHCII, although it has been demonstrated that the majority of pigments of CP29 are more weakly excitonically coupled with one another than in LHCII and may exchange energy due to the Förster mechanism (Cinque et al., 2000).

The individual role of CP29 in the light-harvesting and energy transfer phenomena seems to be to ensure the maximal efficiency of energy trapping by P680 and that of CP26 to optimize the energy flow between LHCII trimers on adjacent PSII-LHCII supercomplexes (Andersson et al., 2001).

PSII particles localized in non-stacked thylakoid membranes $\left(\right.$ PSII $_{\beta}$ ) contain much less LHCII trimers than stacked ones (Jansson et al., 1997; Dekker et al., 2002). It is not clear what is the function of LHCII trimers attached to PSII $_{\beta}$ in stroma lamellae as these particles have been found to be unable to transfer electrons from water to plastoquinone (Albertsson, 1995) and this implies that the excitation energy funneled by LHCII to PSII $_{\beta}$ core complexes is not used to fuel a linear electron transport. PSII particles found in agranal chloroplasts of bundle sheath cells of plants belonging to the NADP-ME subtype of $\mathrm{C}_{4}$ type are partially deficient in LHCII trimers as well (Romanowska et al., 2006). The trimers present serve mainly to transfer the excitiation energy to PSI particles (Pfundel \& Pfeffer, 1997) and this is the reason why the overall electron transporting activity of PSII of bundle sheath cells is much lower than in the case of neighbouring mesophyll cells (Lavergne \& Leci, 1993).
When there is too much light several photoprotective phenomena are switched on at the level of peripheral light-harvesting complexes of PSII in order to lower the risk of photodamage to the photosystem, and thus photoprotection is in addition to the primary role of peripheral light-harvesting complexes as energetic antennae. The photoprotective phenomena occurring at the level of peripheral light-harvesting complexes of PSII are aimed at dissipation of the excessive amount of excitation energy transferred by the complexes and trapped by P680 in order to avoid overreduction of $Q_{A}$ and photodamage of PSII. The excess energy dissipation is catalyzed by a non-photochemical quenching phenomenon which has two components, operating on a time scale of seconds and minutes, respectively. Within several seconds (rapid response) after the exposure to elevated irradiance some thylakoid lumenexposed amino-acid residues of Lhcb4 and Lhcb5 are protonated and this leads to change in their conformation favouring dissipation of energy transfer between pigment molecules instead of conservation (Morosinotto et al., 2003). PsbS may be protonated and significantly involved in the rapid response as well (see paragraph: Pigment-binding proteins of PSII core complex). Within a few minutes (slow response) of exposure to elevated irradiance an acidifaction of the thylakoid lumen promotes the conversion of violaxanthin bound to polypeptides of light-harvesting complexes to zeaxanthin and this triggers the dissipation of an excess of excitation energy. There is a vigorous debate with regard to the exact mode of action of zeaxanhin as a potent quencher of energy transfer and the role of individual peripheral lightharvesting complexes in the slow response. According to the "conformation" model zeaxanthin replaces violaxanthin bound to L2 sites of CP29 and CP26 and acts there to change the conformation of Lhcb4 and Lhcb5, respectively, in a manner favouring dissipation of energy by other pigment molecules attached to Lhcb4/5 instead of transferring it to neighbours (Morosinotto et al., 2003). As mentioned in the paragraph: Pigment-binding proteins of PSII core complex, zeaxanthin most probably binds protonated PsbS as well, and the protonated PsbS-zeaxanthin complex is another important player in the quenching of the excessive amount of excitation energy by the "conformation" mechanism (Aspinal O'Dea et al., 2002; Li et al., 2004). According to a "direct dissipation by zeaxanthin" model zeaxanthin replaces violaxanthin in the L2 site of LHCII apoproteins and serves there to accept energy from one of the chlorophylls and to dissipate it directly as heat (Standfuss et al., 2005).

On a time scale of days lowering of the excess of excitation energy transferred and trapped by P680 is ensured additionally by the reduction of LHCII pool associated with PSII at elevated irradi- 
ance (Larsson et al., 1987) due to downregulation of expression of Lhcb1-3 (both mRNA and apoprotein accumulation is affected - Jansson et al., 2000; Jackowski et al., 2003). In spite of some efforts it is not fully understood which pool of LHCII trimers is absent in plants acclimated to elevated irradiance (Jackowski et al., 2003).

Another physiological function ascribed to LHCII (but not to other peripheral light-harvesting complexes of PSII) is to regulate the distribution of excitation energy between PSII and PSI in response to changes in light quality, i.e. during state transitions. Namely, when irradiance conditions favor photosystem I (PSI) (state 1) LHCII trimers remain attached to the PSII core complex and the excitation energy is evenly distributed between the two photosystems. To keep the balance of excitation of the two photosystems under the irradiance conditions that favour PSII (state 2) a specific pool of LHCII (not identified unequivocally) detaches from PSII core complex, moves laterally within the thylakoid membrane, docks at PSI and donates it with the excitation energy. State transitions are interpreted as light-quality dependent, redox-controlled reversible phosphorylations of the N-termini of Lhcb1 and Lhcb2 leading to detachment form PSII and lateral movement of a specific pool of LHCII between PSII and PSI particles. The sequence of events that occur under state 2 is most probably initiated by activating a kinase - which phosphorylates N-termini of Lhcb1 and Lhcb2 - via reduced plastoquionone bound to the Qo site of cytochrome $b_{6}-f$ complex (Vener et al., 1997). The enzyme responsible was identified in Chlamydomonas reinhardtii as thylakoid membrane-bound, serine-type Stt7 kinase (Depege et al., 2003) and its orthologue STN7 in higher plants (Bellafiore et al., 2005). It is thought that a phosophorylated form of the specific LHCII pool detaches from PSII because it has low affinity for PSII core complex and high affinity for PSI (Allen \& Forsberg, 2001). The phospho-LHCII was found to dock at the PsaH subunit of PSI core (Lunde et al., 2000). It is hypothesized that when the irradiance conditions induce the state 2 - state 1 transition phospho-LHCII is dephosphorylated by the thylakoid membrane-associated phosphatase regulated by cyclophilin-like protein TLP (Carlberg \& Andersson, 1996; Fulgosi et al., 1998), lacks its affinity for PSI and migrates back to PSII. The physiological meaning of these phenomena is to provide preferentially either PSII or PSI with excitation energy delivered by LHCII as to keep the electron flow through photosystems balanced, regardless of the quality of incident light (Gal et al., 1997).

Finally, LHCII is widely regarded to be responsible for thylakoid membrane adhesion and grana formation. This notion is supported by the obser- vations that purified LHCII stimulates grana formation in a model thylakoid membrane system (Mullet \& Arntzen, 1980) and that there is a stringent correlation between the appearance of LHCII in greening plastids and the formation of grana stacks (Anderson et al., 1988). The LHCII-mediated control of thylakoid membrane involves the N-terminal, stroma-exposed region of Lhcb apoproteins by decreasing locally the electrostatic repulsion of approaching thylakoids in the presence of electrostatic screening by cations allowing in this way van der Waals attraction forces between thylakoids to take effect (Mullet \& Arntzen, 1980; Chow et al., 1991). It has been suggested that CP26 and not LHCII may be engaged in promoting the membrane adhesion since barley plants grown under intermittent light conditions and having no detectable amounts of Lhcb type apoproteins due to this - except for Lhcb5 - nonetheless display grana stacks (Król et al., 1995). Furthermore, Lhcb1- and Lhcb2-less Arabidopsis mutants were demonstrated to yield grana stacks too (Andersson et al., 2003). The derived conclusions about the necessity of CP26 and lack of importance of LHCII for grana stacking should be, however, viewed with utmost caution as no precise quantitative processing of micrographs showing thylakoids from mutants, wild type plants and wild type plants grown in intermittent light has been presented.

\section{Degradation of pigment-binding proteins of PSII peripheral light-harvesting complexes}

When a plant grown under medium light conditions encounters high light on a time scale of 24-72 h, LHCII apoproteins are degraded and this phenomenon reflects the strategy of avoiding the donation of an excessive amount of excitation energy to P680 when there is too much light. Namely, a protease belonging to the serine or cysteine class, peripherally attached to the stromal side of the thylakoid membrane, has been shown to cleave preferentially Lhcb 2 in spinach plants acclimated for at least $48 \mathrm{~h}$ to high light (Lindahl et al., 1995). The "acclimative protease", which has not been identified in terms of being a product of a defined gene, degrades selectively Lhcb2-containing LHCII monomers in dephosphorylated form (Yang et al., 1998). An analysis of T-DNA insertion mutants lacking various chloroplast proteases belonging to the $\mathrm{FtsH}$ family demonstrated that FtsH6 - a zinc-dependent metalloprotease, integrally associated with the thylakoid membrane - is responsible for the degradation of Lhcb1 which takes place during 24-72 h-long acclimation of Arabidopsis to high light (Żelisko et al., 2005). The same protease has been found to catalyse the degradation of Lhcb3 during senescence of Arabidopsis leaves. The senescence-related degradation 
of Lhcb3 is an element of a massive turnover of chloroplast macromolecules, marking the transition from the assimiliation phase to the nutrient recycling phase of leaf development (Garcia-Lorenzo et al., 2005; Żelisko et al., 2005). Some other, still unidentified chloroplast protease may be engaged in the degradation of LHCII apoproteins in monomer form during its assembly with pigments in bean etioplasts (Anastassiou \& Argyroudi-Akoyunoglou, 1995; Tziveleka \& Argyroudi-Akoyunoglou, 1998).

No proteases responsible for degradation of apoproteins of minor peripheral PSII light harvesting complexes under changing environmental conditions or in the ontogenetical context have been identified so far.

\section{Acknowledgments}

This work was supported by funds for science in years 2006-2009 as research project N303 061 31/2088 of Polish Ministry of Science and Higher Education.

\section{REFERENCES}

Akabori K, Tsukamoto H, Tsukihara J, Nagatsuka T, Motokawa O, Toyoshima Y (1988) Disintegration and reconstitution of photosystem II reaction center core complex. I. Preparation and characterization of three different types of subcomplexes. Biochim Biophys Acta 932: 345-357.

Allen KD, Staehelin LA (1992) Biochemical characterization of photosystem II antenna polypeptides in grana and stroma membranes of spinach. Plant Physiol 100: 1517-1526.

Allen JF, Forsberg J (2001) Molecular recognition in thylakoid structure and function. Trends Plant Sci 6: 317326.

Anastassiou R, Argyroudi-Akoyunoglou JH (1995) Thylakoid-bound proteolytic activity against LHCII apoprotein in bean. Photosynth Res 43: 241-250.

Anderson JM, Andersson B (1988) The dynamic photosynthetic membrane and regulation of solar energy conversion. Trends Biochem Sci 13: 351-355.

Andreasson E, Svensson P, Weibull C, Albertsson PA (1988) Separation and characterization of stroma and grana membranes - evidence for heterogeneity in antenna size of both photosystem I and photosystem II. Biochim Biophys Acta 936: 339-350.

Anderson J, Walters RG, Horton P, Jansson S (2001) Antisense inhibition of the photosynthetic antenna proteins CP29 and CP26: implications for the mechanism of protective energy dissipation. Plant Cell 13: 1193-1204.

Anderson J, Wentworth M, Walters RG, Howard CA, Ruban AV, Horton P, Jansson S (2003) Absence of Lhcb1 and Lhcb2 proteins of the light-harvesting complex of photosystem II - effects on photosynthesis, grana stacking and fitness. Plant J 35: 350-361.

Anderssson B, Aro E-M (2001) Photodamage and D1 protein turnover in photosystem II. In Advances in Photosynthesis and Respiration - Regulation of Photosynthesis (Aro E-M, Andersson B, eds) vol 11, pp 377-393. Kluwer Academic Publishers, Dordrecht, Boston, London.
Andreucci F, Barbato R, Picollo C, Segalla A (2005) Isolation of phosphorylated and dephosphorylated forms of the CP43 internal antenna of photosystem II in Hordeum vulgare L. J Exp Bot 56: 1239-1244.

Aspinal O'Dea M, Wentworth M, Pascal A, Robert B, Ruban A, Horton P (2002) In vitro reconstitution of the activated zeaxanthin state associated with energy dissipation in plants. Proc Natl Acad Sci USA 99: 16331-1635.

Bailey S, Thompson E, Nixon PJ, Horton P, Mullineaux CW, Robinson C, Mann NH (2002) A critical role for the Var2 FtsH homologue of Arabidopsis thaliana in the photosystem II repair cycle in vivo. ? 277: 2006-2011.

Barber J, Nield J, Morris EP, Zheleva D, Hankamer B (1997) The structure, function and dynamics of photosystem two. Physiol Plant 100: 817-827.

Bassi R, Pineau B, Dainese P, Marquardt J (1993) Carotenoid-binding proteins of photosystem II. Eur J Biochem 212: 297-303.

Bellafiore S, Barneche F, Peltier ZG, Rochaix J-D (2005) State transitions and light adaptation require chloroplast thylakoid protein kinase STN7. Nature 433: 892895.

Boekema EJ, Hankamer D, Bald D, Kruip J, Nield J, Boonstra AF, Barber J, Rögner M (1995) Supramolecular structure of the photosystem II complex from green plants and cyanobacteria. Proc Natl Acad Sci USA 92: 175-179.

Boekema EJ, van Roon H, Calkoen F, Bassi R, Dekker JP (1999) Multiple types of association of photosystem II and its light-harvesting antenna in partially solubilized photosystem II membranes. Biochemistry 38: 2233-2239.

Bricker TM, Frankel LK (1988) The structure and function of $33 \mathrm{kDa}$ extrinsic protein of photosystem II. A critical review. Photosynth Res 56: 157-173.

Bricker TM, Frankel LK (2002) The structure and function of CP47 and CP43 in photosystem II. Photosynth Res 72: 131-146.

Caffari S, Croce R, Breton J, Bassi R (2001a) The major antenna complex of photosystem II has a xanthophyll binding site not involved in light harvesting. J Biol Chem 276: 35924-35933.

Caffari S, Croce R, Cattivelli L, Bassi R (2001b) The Lhcb1, 2, 3 gene products component the trimeric antenna complex of higher plant photosystem II, have distinct biochemical, spectroscopic properties. PS2001 Proceedings 12th International Congress on Photosynthesis. S31034. CSIRO Publishing, Melbourne, Australia.

Cai D, Herrmann RG, Klösgen RB (1993) The 20 kDa apoprotein of the CP24 complex of photosystem II: an alternative model to study import and intra-organellar routing of nuclear-encoded thylakoid proteins. Plant $J$ 3: 383-392.

Callahan FE, Ghirardi ML, Sopory SK, Mehta AM, Edelman M, Mattoo AK (1990) A novel metabolic form of the $32 \mathrm{kDa}-\mathrm{D} 1$ protein in the grana-localized reaction center of photosystem II. J Biochem Sci 265: 1535715360.

Canovas PM, Barber J (1993) Detection of a 10 kDa breakdown product containing the C-terminus of the D1protein in photoinhibited wheat leaves suggests an acceptor side mechanism. FEBS Lett 324: 341-344.

Carlberg I, Andersson B (1996) Phosphatase activities in spinach thylakoid membranes - effectors, regulation and location. Photosynt Res 47: 145-156.

Chow WS, Miller C, Anderson JM (1991) Surface charges, the heterogeneus lateral distribution of the two photosystems, and thylakoid stacking. Biochim Biophys Acta 1057: 69-77. 
Cinque G, Croce R, Holzwarth A, Bassi R (2000) Energy transfer among CP29 chlorophylls: calculated Förster rates and experimetal transient absorption at room temperature. Biophys J 79: 1706-1716.

Croce R, Remelli R, Varotto C, Breton J, Bassi R (1999) The neoxanthin binding sites of the major light harvesting complex (LHCII) from higher plants. FEBS Lett 456: $1-6$.

Dainese P, Hoyer-Hansen G, Bassi R (1990) The resolution of chlorophyll $\mathrm{a} / \mathrm{b}$ binding proteins by a preparative method based on flat bed isoelectro focusing. Photochem Photobiol 51: 511-519.

Deisenhofer J, Epp O, Miki K, Huber R, Michel H (1985) Structure of the protein subunits in the photosynthetic reaction centre of Rhodopseudomonas viridis at $3.8 \AA$ resolution. Nature 318: 618-624.

Dekker JP, van Roon H, Boekema EJ (1999) Heptameric association of light-harvesting complex II trimers in partially solubilized photosystem II membranes. FEBS Lett 449: 211-214.

Dekker JP, Garmano M, van Roon H, Boekema E (2002) Photosystem II solubilizes as a monomer by mild detergent treatment of unstacked thylakoid membranes. Photosynth Res 72: 201-210.

Depege N, Bellafiore S, Rochaix J-D (2003) Role of chloroplast protein kinase Stt7 in LHCII phosphorylation and state transition in Chlamydomonas. Science 299: 15721575.

Dominici P, Caffarri S, Armenante F, Ceoldo S, Crimi M, Bassi R (2002) Biochemical properties of the PsbS subunit of photosystem II either purified from chloroplast or recombinant J Biol Chem 277: 22750-22758.

Dunsmuir P (1985) The petunia chlorophyll a/b binding protein genes: a comparison of Cab genes from different gene families. Nucleic Acids Res 13: 2503-2518.

Eggers B, Vermaas W (1993) Truncation of the D2 protein in Synechocystis sp. PCC 6803: a role of the C-terminal domain of D2 in photosystem II function and stability. Biochemistry 32: 11419-11427.

Eshagi S, Andersson B, Barber J (1999) Isolation of highly active PSII-LHCII supercomplex from thylakoid membranes by a direct method. FEBS Lett 446: 23-26.

Ferreira KN, Iverson TM, Maghlaoui K, Barber J, Iwata S (2004) Architecture of the photosynthetic oxygen-evolving center. Science 303: 1831-1838.

Fulgosi H, Vener AV, Altschmied RG, Herrmann B, Andersson B (1998) A novel multi-functional chloroplast protein: identification of a 40-kDa immunophilin-like protein located in thylakoid lumen. EMBO J 17: 15771587.

Funk C, Schröder WP, Napiwotzki A, Tjus SE, Regner G, Andersson B (1995) The PSII-S protein of higher plants - a new-type of pigment binding protein. Biochemistry 34: 11133-11141.

Gal A, Zer H, Ohad I (1997) Redox-controlled thylakoid protein phosphorylation: news and views. Physiol Plant 100: 869-885.

Garcia-Lorenzo M, Żelisko A, Jackowski G, Funk C (2005) Degradation of the main photosystem II light-harvesting complex. Photochem Photobiol Sci 4: 1065-1071.

Gradinaru CC, Pascal AA, van Mouric F, Robert B, Horton P, van Grondelle R, van Amerongen H (1998) Ultrafast evolution of the excited states in the chlorophyll $\mathrm{a} / \mathrm{b}$ complex CP29 from green plants studied by energyselective pump-probe spectroscopy. Biochemistry 37: 1143-1149.

Gradinaru CC, van Stokkum IHM, Pascal AA, van Grondelle R, van Amerongen $H$ (2000) Identifying the pathways of energy transfer between carotenoids and chlo- rophylls in LHCII and CP29. A Multicolor, Femtosecond Pump-Probe Study. J Phys Chem 104: 9330-9342.

Green BR (1982) Protein synthesis by isolated Acetabularia chloroplasts. Synthesis of the two minor chlorophyll a complexes in vitro. Eur J Biochem 15: 543-546.

Green BR, Pichersky E, Kloppstech K (1991) Chlorophyll a/ b-binding proteins: an extended family. Trends Biochem Sci 16: 181-186.

Greenberg BM, Gaba V, Matto AK, Edelman M (1987) Identification of a primary in vivo degradation product of the rapidly-turning-over 32-kd protein of photosystem II. EMBO J 6: 2865-2869.

Groot ML, Peterman EJG, van Stokkum IHM, Dekker JP, van Grondelle R (1995) Triplet and fluorescing states of the CP47 antenna complex of photosystem II studied as a function of temperature. Biophys J 68: 281-290.

Groot M-L, Frese RN, de Weerd FL, Bromek K, Pettersson A (1999) Spectroscopic properties of the CP43 antenna protein of photosystem II. Biophys J 77: 3328-3340.

Hankamer B, Nield J, Zheleva D, Boekema EJ, Jansson S, Barber J (1997) Isolation and biochemical characterization of monomeric and dimeric photosystem II complexes from spinach and their relevance to the organization of photosystem II iv vivo. Eur J Biochem 243: 422-429.

Hankamer B, Morris E, Nield J, Gerle C, Barber J (2001) Three-dimensional structure of the photosystem II core dimer of higher plants determined by electron microscopy. J Struct Biol 135: 262-269.

Harrer R (2003) Associations between light-harvesting complexes and photosystem II from Marchantia polymorpha L. determined by two- and three-dimensional electron microscopy. Photosynth Res 75: 249-258.

Haussuhl K, Andersson B, Adamska I (2001) A chloroplast DegP2 protease performs the primary cleavage of the photodamaged D1 protein in plant photosystem II. EMBO J 20: 713-722.

Hemelrijk PW, Kwa SLS, van Grondelle R, Dekker JP (1992) Spectroscopic properties of LHCII the main light-harvesting chlorophyll-a/b protein complex from chloroplast membranes. Biochim Biophys Acta 1098: 159166.

Hobe S, Forster R, Klinger J, Paulsen H (1995) N-proximal sequence motif in light-harvesting chlorophyll a/ $\mathrm{b}$ binding protein is essential for the trimerization of light-harvesting chlorophyll $\mathrm{a} / \mathrm{b}$ complex. Biochemistry 34: 10224-10228.

Ikeuchi M, Inoue Y (1988) A new photosystem II reaction center component (4.8-kDa protein) encoded by the chloroplast genome. FEBS Lett 241: 99-104.

Inagaki N, Yamamoto Y, Mori H, Sato K (1996) Carboxylterminal processing peptidase for the D1 precursor protein: cloning and sequencing of the spinach cDNA. Plant Mol Biol 30: 39-50.

Jackowski G, Kluck E (1994) The oligomeric arrangement of the light-harvesting chlorophyll a/b-protein complex of photosystem II. Z Naturforschung 49c: 337-342.

Jackowski G, Przymusiński R (1995) The resolution and biochemical characterization of subcomplexes of the main light-harvesting chlorophyll a/b-protein complex of photosystem II (LHCII). Photosynth Res 43: 41-48.

Jackowski G, Jansson S (1998) Characterization of photosystem II antenna complexes separated by non-denaturing isoelectric focusing. Z Naturforschung 53c: 841-848

Jackowski G, Kacprzak K, Jansson S (2001) Identification of Lhcb1/Lhcb2/Lhcb3 heterotrimers of the main lightharvesting chlorophyll a/b-protein complex of photosystem II (LHCII). Biochim Biophys Acta 1504: 340-345. 
Jackowski G, Olkiewicz P, Zelisko A (2003) The acclimative response of the main light-harvesting chlorophyll $\mathrm{a} / \mathrm{b}$ protein complex of photosystem II (LHCII) to elevated irradiances at the level of trimeric subunits. $I$ Photochem Photobiol B: Biol 70: 163-170.

Jansson S (1994) The light-harvesting chlorophyll a/b-binding proteins. Biochim Biophys Acta 1184: 1-19.

Jansson S (1999) A guide to the Lhc genes and their relatives in Arabidopsis. Trends Plant Sci 4: 236-240.

Kim S, Sandusky P, Bowlby NR, Aebersold R, Green BR, Vlahakis S, Yocum CF, Pichersky E (1992) Characterization of a spinach psbS cDNA encoding the $22 \mathrm{kDa}$ protein of photosystem II. FEBS Lett 314: 67-71.

Koivuniemi A, Aro EM, Andersson B (1995) Degradation of the D1- and D2-proteins of photosystem II in higher plants is regulated by reversible phosphorylation. Biochemistry 34: 16022-16029.

Król M, Spangfort MD, Huner NPA, Öquist G, Gustaffson P, Jansson S (1995) Chlorophyll a/b-binding proteins, pigment conversions, and early light-induced proteins in a chlorophyll b-less barley mutant. Plant Physiol 107: 873-883.

Kuttkat A, Kartmann A, Hobe S, Paulsen H (1996) The C-terminal domain of light-harvesting chlorophyll$\mathrm{a} / \mathrm{b}$-binding protein is involved in the stabilization of trimeric light harvesting complex. Eur J Biochem 242: 288-292.

Kühlbrandt W, Wang DN, Fujiyoshi Y (1994) Atomic model of plant light-harvesting complex by electron crystallography. Nature 367: 614-621.

Larsson UK, Anderson JM, Andersson B (1987) Variations in the relative content of the peripheral and inner lightharvesting chlorophyll a/b-protein complex (LHCII) subpopulations during thylakoid light adaptation and development. Biochim Biophys Acta 894: 69-75.

Lavergne J, Leci E (1993) Properties of inactive photosystem II centers. Photosynth Res 35: 323-343.

Li Z, Burnap RL (2002) Mutations of basic arginine residue 334 in the D1 protein of photosystem II lead to unusual $\mathrm{S}_{2}$ state properties in Synechocystis sp PCC6803. Photosynth Res 72: 191-201.

Li XP, Bjorkman O, Shih C, Grossman AR, Rosenquist M, Jansson S, Niyogi KK (2000) A pigment-binding protein essential for regulation of photosynthetic light harvesting. Nature 403: 391-395.

Li XP, Gilmore AM, Caffarri S, Bassi R, Golan T, Kramer D, Niyogi KK (2004) Regulation of photosynthetic light harvesting involves intrathylakoid lumen $\mathrm{pH}$ sensing by the PsbS protein. J Biol Chem 279: 22866-22874.

Lindahl M, Yang DH, Andersson B (1995) Regulatory proteolysis of the major light-harvesting chlorophyll $\mathrm{a} / \mathrm{b}$ protein of photosystem II by a light-induced membrane-associated enzymic system. Eur I Biochem 231: 503-509.

Lindahl M, Spetea C, Hundal T, Oppenheim AB, Adam Z, Andersson B (2000) The thylakoid FtsH protease plays a role in the light-induced turnover of the photosystem II, D1 protein. Plant Cell 12: 419-431.

Liu Z, Yan H, Wang K, Kuang T, Zhang J, Gui L, An X, Chang W (2004) Crystal structure of spinach major light-harvesting complex at $2.72 \AA$ resolution. Nature 428: 287-292.

Lunde C, Jensen PE, Haldrup A, Knoetzel J, Scheller HV (2001) The PSI-H subunit of photosystem I is essential for state transitions in plant photosynthesis. Nature 408: 613-615.

Michel H, Deisenhofer J (1988) Relevance of the photosynthetic reaction center from purple bacteria to the structure of photosystem II. Biochemistry 27: 1-7.
Morosinotto T, Caffarri S, Dall'Osto L, Bassi R (2003) Mechanistic aspects of the xanthophyll dynamics in higher plant thylakoids. Physiol Plant 119: 347-354.

Morrisey PJ, Glick RE, Melis A (1988) Supramolecular assembly and functions of subunits associated with the chlorophyl a-b light-harvesting complex II (LHCII) in soybean chloroplast. Plant Cell Physiol 30: 335-344.

Mullet JE, Arntzen CJ (1980) Simulation of grana stacking in a model membrane system. Mediation by a purified light-harvesting pigment-protein complex from chloroplasts. Biochim Biophys Acta 589: 100-117.

Nanba O, Satoh K (1987) Isolation of a photosystem II reaction center consisting of D-1 and D-2 polypeptides and cytochrome b-559. Proc Natl Acad Sci USA 84: 109-112.

Nield J, Kruse J, Ruprecht P, da Fonseca P, Büchel C, Barber J (2000) Three-dimensional structure of Chlamydomonas reinhardtii and Synechoccoccus elongatus photosystem II complexes allows for comparison of their oxygen-evolving complex organization. J Biol Chem 275: 27940-27946.

Paulsen H (1995) Chlorophyll a/b binding proteins. Photochem Photobiol 62: 376-382.

Petersen J, Dekker JP, Bowlby NR, Ghanotakis DF, Yocum CF, Babcock GT (1990) EPR characterization of the CP47-D1-D2-cytochrom b559 complex of photosystem II. Biochemistry 29: 3226-3231.

Pfundel E, Pfeffer M (1997) Modification of photosystem I light harvesting of bundle-sheath chloroplasts occurred during the evolution of NADP-malic enzyme C4 photosynthesis. Plant Physiol 114: 145-152.

Remelli R, Varotto C, Sandona D, Croce R, Bassi R (1999) Chlorophyll binding to monomeric light-harvesting complexes. A mutation analysis of chromophore-binding residues. J Biol Chem 274: 33510-33521.

Rhee K-H, Morris EP, Barber J, Kühlbrandt W (1998) Threedimensional structure of the plant photosystem II reaction centre at $8 \AA$ resolution. Nature 396: 283-286.

Rogl H, Schodel R, Lokstein H, Kuhlbrandt W, Schubert A (2002) Assignment of spectral substructures to pigment-binding sites in higher plant light-harvesting complex LHC-II. Biochemistry 41: 2281-2287.

Rogner R, Chrisholm DA, Diner DA (1991) Site-directed mutagenesis of the PsbC gene of photosystem II: isolation and functional characterization of CP43-less photosystem II core complex. Biochemistry 30: 5387-5395.

Romanowska E, Drożak A, Pokorska B, Shiell B, Michalski WP (2006) Organization and activity of photosystems in the mesophyll and bundle sheath chloroplasts of maize. J Plant Physiol 163: 607-618.

Ruban AV, Wentworth M, Yakushevska AE, Andersson J, Lee PJ, Keegstra W, Dekker JP, Boekema EJ, Jansson S, Horton P (2003) Plants lacking the main light-harvesting complex retain photosystem II macro-organization. Nature 421: 648-652.

Sakamoto W, Tamura T, Hanba-Tomita Y, Murata M, Sodmergen (2002) The VAR1 locus of Arabidopsis encodes a chloroplastic FtsH and is responsible for leaf variegatioin in the mutant alleles. Genes Cells 7: 769-780.

Sandona D, Croce R, Pagano A, Crimi M, Bassi R (1998) Higher plants light harvesting proteins. Structure and function as revealed by mutation analysis of either protein or chromophore moieties. Biochim Biophys Acta 1365: 207-214.

Stewart DH, Brudvig GW (1998) Cytochrome $b_{559}$ of photosystem II. Biochim Biophys Acta 1367: 63-87.

Standfuss J, Kühlbrandt W (2004) The three isoforms of the light-harvesting complex II. J Biol Chem 279: 3688436891. 
Standfuss J, Terwisscha van Scheltinga AC, Lamborghini M, Kühlbrandt W (2005) Mechanism of photoprotection and nonphotochemical quenching in pea light-harvesting complex at $2.5 \AA$ resolution. EMBO J 24: 919-928.

Šetlik I, Allakhverdiev SI, Nedbal L, Šetlikova E, Klimov VV (1990) Three types of photosystem II photoinactivation. I. Damaging processes of on the acceptor side. Photosynth Res 23: 39-48.

Telfer A, Bishop SM, Phillips D, Barber J (1994) Isolated photosynthetic reaction center of photosystem II as a sensitizer for the formation of singlet oxygen. Detection and quantum yield determination using a chemical trapping technique. J Biochem Sci 269: 13244-13253.

Tziveleka LA, Argyroudi-Akoyunoglou JH (1998) Implications of a developmental-stage-dependent thylakoidbound protease in the stabilization of the light-harvesting pigment-protein complex serving photosystem II during thylakoid biogenesis in red kidney bean. Plant Physiol 117: 961-970.

Vass I, Styring S, Hundal T, Koivuniemi A, Aro E, Andersson B (1992) Reversible and irreversible intermediates during photoinhibition of photosystem II: stable reduced QA species promote chlorophyll triplet formation. Proc Natl Acad Sci USA 89: 1408-1412.

Vener AV, van Kan PJM, Rich PR, Ohad I, Andersson B (1997) Plastoquinol at the quinol oxidation site of reduced cytochrome bf mediates signal transduction between light and protein phosphorylation: thylakoid protein kinase deactivation by a single turnover flash. Proc Natl Acad Sci USA 94: 1585-1590.

Wollman FA, Minai L, Nechtushtai R (1999) The biogenesis and assembly of photosynthetic proteins in thylakoid membranes. Biochim Biophys Acta 1411: 21-85.
Yakushevska AE, Jensen PE, Keegstra W, van Room $\mathrm{H}$, Scheller HV, Boekema EJ, Dekker JP (2001) Supramolecular organization of photosystem II and its associated light-harvesting antenna in Arabidopsis thaliana. Eur J Biochem 268: 6020-6028.

Yakushevska AE, Keegstra W, Boekema EJ, Dekker JP, Andersson J, Jansson S, Ruban AV, Horton P (2003) The structure of photosystem II in Arabidopsis: Localization of the CP26 and CP29 antenna complexes. Biochemistry 42: 608-613.

Yamamoto Y (2001) Quality control of photosystem II. Plant Cell Physiol 42: 121-128.

Yang D-H, Webster J, Adam Z, Lindahl M, Andersson B (1998) Induction of acclimative proteolysis of the lightharvesting chlorophyll $\mathrm{a} / \mathrm{b}$ protein of photosystem II in response to elevated light intensities. Plant Physiol 118: 827-834.

Zolla L, Timperio AM, Walcher W, Huber CG (2003) Proteomics of light-harvesting proteins in different plant species. Analysis and comparison by liquid chromatography-electrospray ionization mass spectrometry. Photosystem II. Plant Physiol 131: 198-214.

Zouni HT, Witt J, Kern P, Fromme N, Krauss N, Saenger W, Orth P (2001) Crystal structure of photosystem II from Synechococcus elongatus at $3.8 \AA$ resolution. Nature 409: 739-743.

Żelisko A, Garcia-Lorenzo M, Jackowski G, Jansson S, Funk C (2005) AtFtsH6 is involved in the degradation of the light-harvesting complex during high-light acclimation and senescence. Proc Natl Acad Sci USA 102: 13699-13704. 